\title{
Comments on Editorial: Has Noninvasive Prenatal Testing (NIPT) Come of Age?
}

\author{
Chanchal Singh $^{1} \cdot$ Anita Kaul ${ }^{2}$
}

Received: 8 August 2015/Accepted: 10 August 2015/Published online: 8 September 2015

(C) Federation of Obstetric \& Gynecological Societies of India 2015

Dear Editors,

The editorial on non-invasive prenatal testing (NIPT) published in the May-June, 2015 issue comes at a very opportune time, and we must applaud the author for the enormous effort it takes to plow through the rapidly expanding evidence and present it in a concise manner.

We feel that certain points need to be highlighted, which may be helpful in decision making in daily clinical practice.

First, NIPT or cell-free fetal DNA (cffDNA) is undoubtedly the best screening test available for prenatal detection of trisomy 21 in singleton pregnancy. The latest meta-analysis by Gil et al. [1] reports a weighted pooled detection rate (DR) of $99.2 \%$ and a false positive rate (FPR) of $0.09 \%$. Most studies available so far and thus quoted in the editorial included high-risk women in which a screening test would indeed perform better. A recently published large, multicentric trial has reported higher

Chanchal Singh is a Consultant, Fetal Medicine, and Anita Kaul is a Senior Consultant and Head in Indraprastha Apollo Hospitals.

Chanchal Singh

chanchalsngh@gmail.com

1 Apollo Centre for Fetal Medicine, Indraprastha Apollo Hospitals, D 701, Kaveri Apartments, Alaknanda, New Delhi 110076, India

2 Apollo Centre for Fetal Medicine, Indraprastha Apollo Hospital, New Delhi 110076, India sensitivity (100\% DR), lower FPR (0.06\%), and higher PPV (80.9\%) for cffDNA testing for trisomy 21 compared to standard screening with first trimester nuchal translucency and biochemistry in routine prenatal screening population [2]. Thus, cffDNA is indeed the best screening test for trisomy 21 even in low-risk population.

An important factor in maintaining the high DR is fetal fraction ( $\geq 4 \%$ for most labs). Thus clinicians must insist that fetal fraction is provided with results. If the test cannot be reported due to low fetal fraction, the possibility of aneuploidy is higher [2] and invasive testing viz a viz repeat sampling should be discussed. Fetal fraction is also likely to be low in women with high BMI and these women should be counseled accordingly. Fetal fraction is sufficient for reporting after 10 weeks gestation. The turnaround time for cfDNA is 7-10 days; thus gestational age will be important in deciding for the test as one would like to have a result well before 20 weeks to allow time for invasive testing if needed.

cffDNA is still a 'screening test' and the decision to terminate must NOT be based on it. All high-risk/positive results must be offered invasive testing to confirm (or indeed rule out) aneuploidy. This should preferably be by amniocentesis to minimize errors due to placental mosaicism [4].

Since its role in multiple pregnancies is still being validated, cffDNA is not yet recommended for these (including those with an empty sac and/or vanishing twin) $[1,3]$. 
cffDNA is NOT an alternative to first trimester screening that can easily pick up structural abnormalities which are more common than chromosomal abnormalities [1, 3, 5]. The advantages of early diagnosis of a structural abnormality in terms of patient counseling and ease of early termination (in case the abnormality warrants it) cannot be over emphasized. In addition, the combined test also screens for obstetric complications like preeclampsia and fetal growth restriction apart from correctly dating the pregnancy.

Finally, since cfDNA tests only for limited chromosomes, it should not be offered to women with increased NT or an obvious structural abnormality on ultrasound [3].

We feel that the most economical way of implementing this costly test would be in a contingent manner: all women should be offered combined first trimester screening by ultrasound, PAPP-A, and betahCG. Women with very high risk ( $\geq 1: 10$ ) should be offered invasive testing, whereas women with intermediate risk (1:11-1:2500) should be offered cffDNA. This policy would require cfDNA for $1 / 4$ th of the screened population and detect $98 \%$ of trisomies at an invasive rate of only $0.8 \%$ [5].

With the explosion of information on internet and aggressive marketing by companies facilitating cffDNA, we are bound to think about an optimal strategy for its clinical application and counsel our patients appropriately.
Funding No source of funding.

\section{Compliance with Ethical Standards}

Conflict of interest The authors declare there is no conflict of interest, and there are no financial disclosures to be made.

\section{References}

1. Gil MM, Quezada MS, Revello R, et al. Analysis of cell-free DNA in maternal blood in screening for fetal aneuploidies: updated meta-analysis. Ultrasound Obstet Gynecol. 2015;45:249-66.

2. Norton ME, Jacobsson B, Swamy GK, et al. Cell-free DNA analysis for noninvasive examination of trisomy. N Engl J Med. 2015;372(17):1589-97.

3. Committee opinion no 640: cell free DNA screening for aneuploidies. American College of Obstetricians and Gynecologists Committee on Genetics. Obstet Gynecol. 2015, Jun 29. [Epub ahead of print].

4. Everett TR, Chitty LS. Cell-free fetal DNA: the new tool in fetal medicine. Ultrasound Obstet Gynecol. 2015;45(5):499-507.

5. Gil MM, Akolekar R, Quezada MS, et al. Analysis of cell-free DNA in maternal blood in screening for aneuploidies: metaanalysis. Fetal Diagn Ther. 2014;35:156-73. 